\title{
Téoros
}

Revue de recherche en tourisme

\section{Les dimensions économiques de l'aménagement touristique}

\section{Paul Bodson et Jean Stafford}

Volume 2, numéro 3, octobre 1983

Les multiples facettes de l'aménagement touristique

URI : https://id.erudit.org/iderudit/1080827ar

DOI : https://doi.org/10.7202/1080827ar

Aller au sommaire du numéro

Éditeur(s)

Université du Québec à Montréal

ISSN

0712-8657 (imprimé)

1923-2705 (numérique)

Découvrir la revue

Citer cet article

Bodson, P. \& Stafford, J. (1983). Les dimensions économiques de l'aménagement touristique. Téoros, 2(3), 20-23. https://doi.org/10.7202/1080827ar d'utilisation que vous pouvez consulter en ligne.

https://apropos.erudit.org/fr/usagers/politique-dutilisation/ 


\title{
Les dimensions économiques de l'aménagement touristique
}

\author{
Par Paul Bodson et Jean Stafford
}

La place réservé au tourisme dans l'orga nisation et l'aménagement d'une région résulte d'un jugement de valeur porté sur la capacité de cette activité de contribuer au développement et à l'intégration de la région. Ce jugement s'articule sur les caractéristiques spécifiques de l'activité de tourisme, sur ses interactions avec les autres activités économiques, sur ses retombées au niveau du bien-être et du mode de vie des populations locales.

Ce texte se propose, à partir des composantes constitutives de l'activité de tourisme, de dégager la nécessité d'un aménagement règional raisonné et de définir les orientations majeures qui en résultent. L'analyse économique du tourisme, au niveau régional, doit tenir compte:

- de la lutte pour l'espace qu'implique la fonction touristique:

du personnel utilisé dans l'industrie touristique:

de l'effet multiplicateur du tourisme;

de la saisonnalité:

et, enfin, de la place du tourisme dans l'ensemble des activités économiques de la région considérée.

Le tourisme dans la lutte pour l'espace régional

Le tourisme régional attire sur un temps rela tivement court des populations, extérieures à la région, intéressées à tirer un plaisir individuel des atouts de la région.

Les activités rattachées au tourisme sont diverses. Elles peuvent relever du contexte géographique (paysage, climat...), des activités greffees sur le contexte géographique (par exemple, les activités sportives issues de la présence d'un lac, d'une rivière ou d'un terrain accidenté), de l'insertion humaine dans le cadre géographique (urbanisation plus ou moins typique; repère historiquel et du contexte culturel. Quelles qu'elles soient, ces différentes activités présentent un caractère commun: celui de toucher un aspect relevant du plaisir personnel de la personne pratiquant cette activité. Une consequence immédiate en découle pour l'aménagement d'une région. Si l'aménagemnet d'une région prévilégie le tourisme, il s'agira d'en promouvoir la qualité. Cette qualité est menacé à deux niveaux. Lorsqu'une région dispose d'atouts touristiques spécifiques, il se développera un certain nombre d'initiatives individuelles structurées par la recherche du profit. Ces initiatives tirent avantage des attraits de la région mais négligent en même temps les répercussions provoquées sur l'environnement. II n'est pas garanti, non plus, que les initiatives individuelles n'orientent les activités touristiques dans une optique de consommation uniformisante, dété riorant ainsi les potentialités initiales. $\mathrm{A}$ un second niveau, les attraits touristiques d'une région peuvent être dégradés par les autres activités économiques. Ce genre de conflit ne trouve généralement pas une solution spontanée.

D'autres conflits peuvent aussi survenir; la fonction récréative, qui se rapporte à l'utilisation par des régionaux des sites, peut par exemple entrer en concurrence avec la fonc tion touristique, qui désigne l'utilisation de ces mèmes sites par des non-régionaux. ${ }^{(1)}$

La lutte pour l'espace régional que se livrent la fonction touristique et les autres fonctions implique des retombees négatives. L'opposition entre la fonction touristique et la fonction récréative peut freiner le développement du tourisme régional; de la même façon, l'opposition latente entre la fonction touristique et la protection de l'environnement ${ }^{|2|}$ peut entrainer, a long terme, des coúts très élevés. Enfin, le conflit entre la fonction touristique et la fonction industrielle peut dégrader jusqu'à le rendre inutilisable, le potentiel touristique d'une région.

L'étude des dimensions économiques de l'aménagement touristique devra dès lors proposer une certaine equilibration des diverses fonctions. ll faudra développer une rationalisation des activités économiques respectant l'importance accor dée à chaque activité et ménageant des solutions les moins conflictuelles possibles.
Le personnel utilisé dans les activités touristiques

Les activités touristiques valorisent les avantages naturels ou acquis par l'implantation humaine en y associant une main d'oeuvre et une infrastructure d'équipement. L'infras tructure d'équipement est indispensable sous peine de maintenir stagnantes les potentialités de la région. Par contre, l'équipement à lui seul, est incapable de valoriser le potentiel touristique d'une région. Le tourisme est une activité tertiaire à utilisation élevée de personnel. Par le fait même que les activités touristiques touchent un noyau où la qualité du plaisir est essentielle, les competences professionnelles et humaines du personnel oeuvrant dans les activités touristiques prennent une grande importance. A ce niveau aussi, une attention particulière des personnes responsables de l'amenagement régional s'impose. II existe des modes d'implantation touristique géné ralement à dimension industrielle, où les ressources régionales de main-d'oeuvre peuvent ètre sous-utilisées. On confinera, par exemple, cette main-d'oeuvre dans des rôles subalternes d'exécution, tandis que leur echappera l'organisation interne de l'activité touristique. Ce faisant, on finit par ignorer l'apport original au tourisme que comporte l'insertion d'une population dans une région.

L'effet multiplicateur du tourisme au niveau régional

En étant orienté vers des services offerts à des personnes venues de l'extérieur, l'activité touristique se définit comme une activité essentiellement exportatrice. Comme toute activité exportatrice, elle gènère un certain revenu dans la région, lequel à son tour, génère des dépenses et des activités sources de revenu. II est donc possible, du moins théoriquement, d'admettre un effet multiplicateur de cette activité sur la région ${ }^{(3)}$. En fait, l'information en termes de revenus et de depenses de consommation, est souvent lacunaire au niveau regional. Par contre, il est possible d'en établir un certain substitut en termes d'emploi. Etant donné l'emploi existant dans les activités d'expor. tation, dans le cas présent les activités tou ristiques, on se demande quel emploi total 
est généré dans la région. Le surplus d'emploi, par rapport aux activités spécifiquement exportatrices, est dú aux activités requises par les activités exportatrices et par les services à la population.

II existe plusieurs procédures pour apprécier le multiplicateur d'emploi touristique.

Selon Pierre Defert ${ }^{(4)}$ I'approximation du nombre d'emplois (directs et indirectsi induits par le tourisme, au niveau régional, peut être faite de deux manières.

1. par la proportionalité régionale à l'aide de la formule:

$$
E_{t}=\frac{E \times P_{t}}{P_{r}} \text { où }
$$

$E_{1}=$ le nombre diemplois induits par le tourisme:

$E=$ le nombre d'emplois total de la region:

$\mathrm{P}_{\mathrm{f}}=$ le produit régional total en termes de valeur ajoutée, issu de l'ensemble des activites economiques:

$\mathrm{P}_{\mathrm{t}}=$ le produit touristique.

2. Par la proportionalité par branche; celleci est établie en fonction de la totalité des emplois touristiques induits dans chacune des branches de la comptabilité régionale:

$E_{t}=\sum_{1}^{N} E_{i t}$ :

où $E_{i t}$ désigne le nombre d'emplois induits par le tourisme (t) dans la branche (ii).

Eit est approximé par l'expression;

$E_{i t}=\frac{E_{i} \times P_{i}}{T}$

dans laquelle $\mathrm{P}$ désigne le produit de la branche i et $T$ la valeur ajoutée du tourisme.

II est possible d'adapter cette seconde méthode pour tenir compte du caractère saisonnier des activités touristiques ${ }^{(5)}$.

Le multiplicateur d'emploi, en tant que subs titut du multiplicateur de revenu, est criticable du fait que l'emploi est toujours associé à un capital. Par contre, dans le cas du tourisme, la critique est moins déterminante en raison de l'importance en qualité et en quantité que prend le personnel.

Dans le cas du tourisme peut-on s'attendre a un effet multiplicateur important? A priori, l'effet multiplicateur semble insensible au type d'activité exportatrice. Par contre, la réalité semble beaucoup plus complexe. Très souvent, l'activité touristique implique une main-d'oeuvre qui aurait été contrainte d'émigrer en l'absence de cette activité. En ce sens, l'activité touristique contribue indubitablement à stabiliser la population. L'effet multiplicateur semble donc rél et effectif.
Par contre, l'activité touristique est le plus: généralement saisonniëre. Si les variations saisonnières sont trop marquées, il en résultera une fuite de population. II n'est même pas exclu que la main-d'oeuvre saisonnière vienne de l'extérieur. A ce moment, l'effet multiplicateur est neutralisé. On voit à ce niveau l'importance d'une planification regionale du tourisme. Si les activités touristiques $n$ "arrivent pas à se diversifier pour atténuer les mouvements saisonniers, si les activites de support $s$ implantent systema tiquement en dehors de la région. l'effet multiplicateur risque de disparaitre et les bénéfices du tourisme régional risquent d'echapper aux populations locales.

L'effet multiplicateur est aussi relie, à la capacité de développer le secteur tertiaire ${ }^{*}$; selon Mario Polèse de I'I.N.R.S.: "Le role moteur des activités tertiaires ne paraît nulle part plus évident que dans le domaine de l'économie urbaine et regionalem7n. Le même auteur définit comme étant "motrice": "Toute activité tertiaire qui s'exporte ou qui contribue a la capacité actuelle et future de production $^{\prime 18}$. Le développement touristique peut être soit la cause, soit l'effet de l'accroissement du tertiaire; les deux eléments sont fortement corrélés au niveau régional.

II existe une véritable hiérarchie du secteur tertiaire: le schérna I, ci-dessous, illustre bien ce phènomène:
On voit qu'à un haut niveau de developpement touristique correspond un niveau elevé de tertiarisation des activités économiques.

Pierre Defert a formule ce qu'il appelle "le quotient touristique d'activite tertiaire ${ }^{m(9)}$. Le QTAT correspond au rapport du nombre d'activités économiques sous influence directe du tourisme régional à la population normale; il s'écrit:

QTAT $=\frac{P}{\text { NET }}$

Selon lui: "Ce taux agit comme un révella. teur, car il explique que certains commerces se maintiennent dans une position marginale grace a lappoint saisonnier du tourisme et disparaitraient autrement. Si fon renforce leur position en développant le tourisme, leur position se consolidera très vite et ils feront tres volontiers les efforts d'auto-financement nécessaires " ${ }^{210}$.

Dans l'ensemble, plus le tertiaire est elevé et hiérarchisé, plus la fonction touristique est intégrée à la région et plus le multiplicateur est ellevé.

L'estimation des effets economiques du tourisme doit aussi prendre en consideration d'autres éléments. II est fréquent par exemple que les activités de support requises au bon déroulement du tourisme lla fabrication de matériel est un cas typiquel se situe a l'extérieur de la région. Cette situation ralen. tit en pratique l'effet multiplicateur.

Le tourisme: une activité saisonnière Le tourisme en général est très dépendant de la saisonnalité: si on considere le tableau $\mathrm{I}$, on voit combien le tourisme au Canada est tributaire des saisons.

\section{Schéma I}

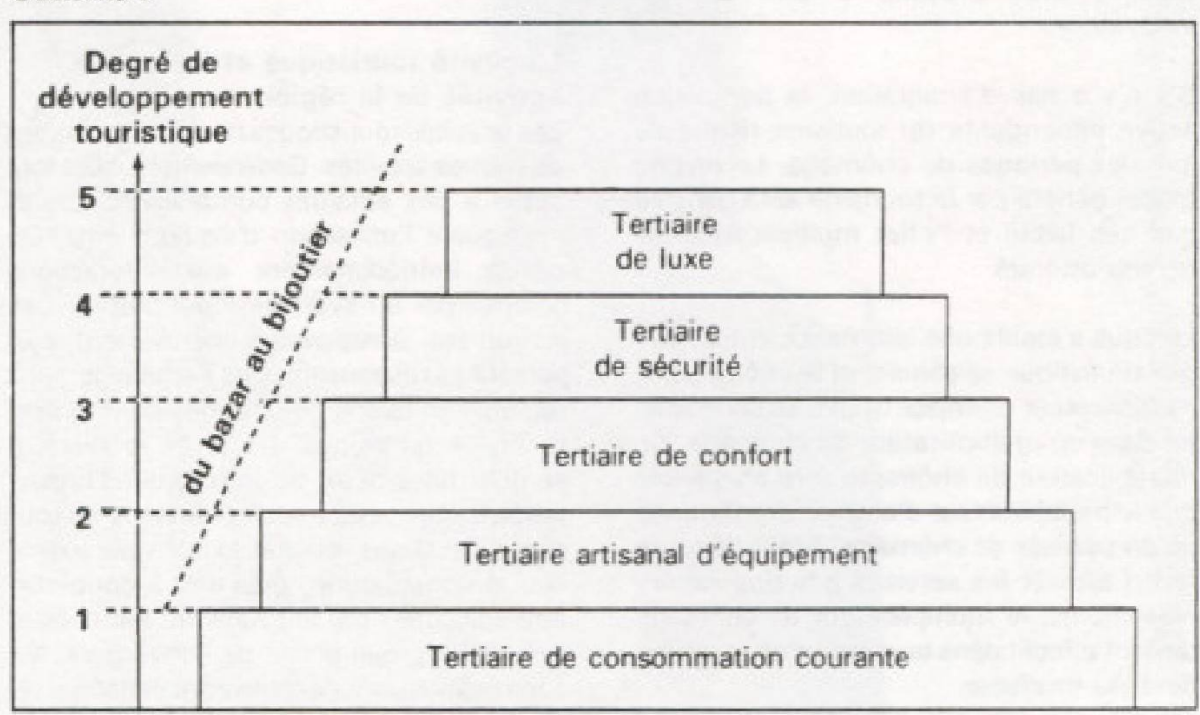

Tiré de: DEFERT, P., "Le tourisme facteur de valorisation régionale", in Recherche Sociale, no 3, Paris, janvier 1966, p. 21. 
Tableau I:

Comparaison des coéfficients saisonniers de six provinces canadiennes â partir des entrées des voyageurs non résidents des années 1977-1981

\begin{tabular}{lcccr}
\hline Provinces & \multicolumn{4}{c}{ Coefficients saisonniers selon les trimestres } \\
& 1 & 2 & 3 & 4 \\
\hline Nouvelle Ecosse & 17 & 93 & 343 & 48 \\
Nouveau Brunswick & 72 & 103 & 146 & 84 \\
Quebec & 64 & 101 & 156 & 78 \\
Ontario & 67 & 116 & 178 & 76 \\
Manitoba & 67 & 150 & 220 & 81 \\
Alberta & 36 & 194 & 247 & 61
\end{tabular}

Sources: Calculs sommaires des auteurs d'après les données de Statistiques Canada.

Au niveau régional, cette dépendance visavis des contraintes climatiques est encore plus importante; l'aménagement des sta. tions de ski, par exemple, doit tenir compte de facteurs physiques très particuliers ${ }^{11 !}$.

Les implications des variations saisonnières sont multiples. La période de gain étant rela tivement limitée, les prix des activités tou. ristiques risquent d'être majorés. Cette réaction est le reflet du jeu du marché. II faut néanmoins souligner le caractère aveugle de ce mécanisme et la possibilité de débloquer cette situation de rareté de revenu par le développement d'activités complémentaires susceptibles d'attenuer l'impact saisonnier. ${ }^{(12)}$

Confrontées aux variations saisonnières, les populations plus ou moins directement liées a l'activité touristique vont réagir de façons diverses. La manière extrême de réagir est de refuser le contre-coup des changements saisonniers en émigrant. II y a dans ce cas dépérissement progressif de la région. Le phénomène sera d'autant plus accentué que la population émigrante est généralement la population jeune, c'est-à-dire la population la plus propice à une activité innovatrice.

S'il n'y a pas d'émigration, la population active dépendante du tourisme risque de longues périodes de chómage. Le revenu global généré par le tourisme sera dans ce cas très faible et l'effet multiplicateur de revenu atténué.

Lorsque s'établit une alternance entre l'em. ploi touristique saisonnier et le chômage, le multiplicateur d'emploi trouve sa contrepar. tie dans un multiplicateur de chômage. Ce multiplicateur de chômage sera plus faible que le multiplicateur d'emploi, étant donné qu'en période de chômage, il faut de toute façon assurer les services à la population. Néanmoins, le multiplicateur de chômage devient effectif dans tous les emplois dépendant du tourisme.

La solution à ce type de problème réside dans une planification des activites touristiques. II importe de creer des activités com.

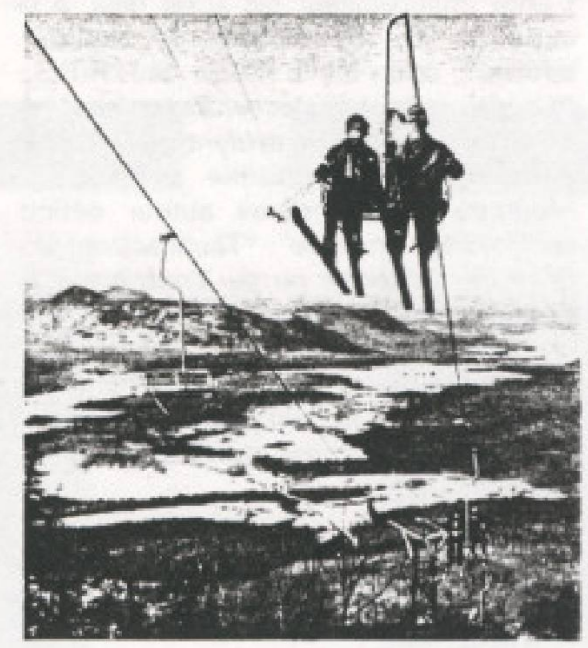

Le tourisme: une activité saisonnière... L'amé nagement des stations de ski doit tenir compte de facteurs physiques tres particuliers.

plémentaires, aptes à atténuer les refiux saisonniers, sous peine de provoquer un chomage endémique, et un niveau de revenu générateur de stagnation et d'appauvrissement de la région.

\section{L'activité touristique et les autres activités de la région}

Les activites touristiques ne sont pas isolées des autres activités. Généralement, elles font appel à des activités complémentaires et impliquent I'utilisation d'equipements. On pense immédiatement aux interactions décrites par un système input-output. Cet instrument échappera généralement aux personnes responsables de l'aménagement régional en raison des lacunes dans l'information economique. De toute manière, il semble hasardeux de surévaluer l'importance des liens intersectoriels issus du tourisme. Certaines activites locales, par exemble la construction, peuvent, à coup sûr, être sollicitées par le tourisme. Par contre, dès qu'un projet prend de l'envergure, les soumissions vont partieliement echapper au réseau strictement régional. II en est de mème de l'équipement. Un equipement relativement spécialisé sera généralement fabriqué à l'extérieur de la région. L'interdé pendance sectorielle sera dès lors très fai" ble. II dépend néanmoins des autorités régionales de valoriser les atouts régionaux pour retenir au maximum dans la région les activités complémentaires au tourisme. Sou lignons enfin, qu'en dépit de liens intersectoriels relativement faibles, l'essor d'une activité touristique dans une région demeure bènéfique par ses retombées en termes de revenus et de taxes. II revient des lors aux autorités régionales d'en promouvoir le développement.

L'activité touristique risque aussi d'entrer en conflit avec d'autres activités économiques. L'activitè touristique de qualité exige géné ralement un niveau élevé de respect de l'environnement que supporte difficilement le développement des activités industrielles. Spontanement, le conflit se résoud par un rapport de forces. S'il est possible, et c'est généralement le cas, de trouver une autre solution, elle résultera d'une harmonisation planifièe.

Face aux autres activites économiques régionales, l'aménagement touristique suppose dès lors une série de choix souvent difficiles. La première étape dans cette démarche consiste à cerner le caractère attractif de la région ou plus specifiquement d'un certain nombre de sites de cette mème région à partir des flux touristiques. Les modèles de "gravitation-inertie" permettent (même s'ils ont des limites importantes) de se faire une certaine idée de la vocation tou. ristique d'une région ou d'un site. Dans une forme générale et simplifiée les principaux

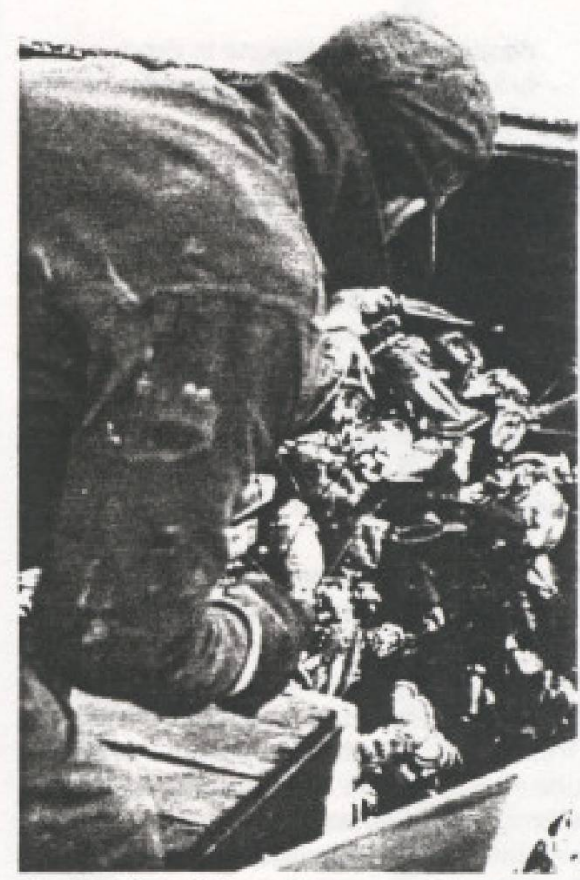

L'activité touristique risque aussi d'entrer en conflit avec d'autres activités économiques... 
modeles de gravitation-inertie sont formulés de la façon suivante:

$N i j=b_{1} \quad \frac{P_{i}^{b_{2}}}{T_{i j} b_{3}} ;$ où

Nij = le nombre de nuitées dans la zone " $j$ " pour un type d'hébergement donné concernant des visiteurs en provenance de la zone émettrice i:

$\mathrm{Pi}=$ la population de la zone émettrice i:

Tij = la distance entre la zone i et la zone j:

$b_{1}, b_{2}, b_{3}=$ les paramètres estimés à partir des donnees; $b_{3}$ traduit la friction spatiale: plus la valeur de $b_{3}$ est élevée, plus l'effet négatif de la distance est grand ${ }^{113}$

La deuxième étape cherche à éclairer les choix a faire entre plusieurs types d'activites economiques touristiques et nontouristiques). La programmation linéaire appliquée au tourisme peut contribuer à donner une image globale des coúts selon que l'on adopte telle ou telle activite ou l'une ou l'autre forme d'hébergement ou l'un ou l'autre type d'équipement; la méthode permet de tenir compte des interactions entre un certain nombre de variables et de simuler des situations hypothétiques, de manière à choisir la solution la plus avantageuse ${ }^{174 !}$.

La troisième étape consiste à établir le mieux possible les benéfices et les coûts qu'entrainent un projet d'aménagement touristique; il s'agit, en quelque sorte, d'établir la rentabilite économique et sociale de ce projet tour ristique au plan régional. Les études de ren. tabilité economique font appel, le plus souvent, au calcul d'actualisation; celui-ci "nous permet de ponderer la valeur de flux monétaires en fonction de lannee ou ils apparaissent, donc d'apprecier de facon plus précise la valeur actuelle des flux de coûts et de recettes apparaissant sur une longue periode 415 ?

L'analyse avantages-coûts tentera d'elargir l'évaluation qu'elle propose aux eléments du projet étudié qui ne sont pas strictement monetaires; elle donnera une image plus globale, plus large de la situation car: "Elle se place du point de vue de la collectivité (locale, provinciale, nationale) pour estimer les coüts et les avantages d'un projet "Allibl. Des travaux ont dejà été entrepris selon cette approche dans le domaine du tourisme régional ${ }^{(17)}$

La quatrième et dernière étape consiste à élaborer des prévisions len incluant les études dejà réaliséesl; elle constitue l'achèvement des recherches des differents impacts d'un projet touristique dans une région donnée ${ }^{\text {nal }}$. Chacune de ces étapes va ser. vir à accroitre les connaissances sur l'environnement régional et favoriser une meilleure prise de décision.

\section{Conclusion}

L'étude des dimensions économiques de l'aménagement régional suppose une prise de conscience de l'aspect conflictuel lié à la lutte pour l'espace entre résidents/nonrésidents d'une part, entre exploitants/nonexploitants d'autre part; il y aura toujours une tension entre les intérêts visant l'exploitation des ressources et les intérèts pour la préservation des mêmes ressources. L'amenagement touristique devra maintenir un certain équilibre dans ce champ clos.

L'amenagement touristique doit veiller à l'insertion des collectivités locales aux projets. touristiques: la main-d'oeuvre issue du milieu va permettre une mise en valeur de la région sur divers plans:

gräce à ses connaissances des differents environnements (sites naturels, patrimoine, cuisine, etc...

par une meilleure utilisation des ressources humaines:

enfin en donnant "une couleur locale" à l'hospitalité régionale.

Le développement touristique passe par le développement du secteur tertiaire. Le tourisme peut, à certaines conditions, devenir "une activité motrice" participant à une croissance régionale harmonieuse.
Le tourisme en général et le tourisme régional en particulier sont fortement marqués par la saisonnalité. Le maintien des personnes sur place et la rentabilisation des equipements supposent que les revenus du tourisme atteignent un certain "seuil critique". Les problèmes inherents à la saisonnalité ne seront réglés que dans le long terme.

Par rapport à l'ensemble des activités économiques régionales, l'industrie touristique va occuper une place à part. Son développement incontrölé risque de conduire à une devalorisation du potentiel touristique d'une région, à une sous-utilisation qualitative et quantitative de la main d'oeuvre, à un chömage saisonnier endémique, de même qu'a une fuite importante de revenus dont pourrait profiter la région.

Par contre, I'aménagement rationnel et harmonieux du tourisme peut être un facteur de progrès au niveau régional en permet. tant, par la prise de conscience de l'environnement naturel, une exploitation plus humaine et, dès lors plus efficace, des atouts de la région. Ce type d'aménagement pourrait aussi avoir un effet d'entrainement sur la facon de percevoir et d'orienter l'ensemble du développement régional. $f$

\section{Références}

1. MESPUER, I. L amenagement touristique difficul. tés de definition ou ditierence de conceprion? in Revue d'Economie Regionale et Urbaine. no 1. Paris, 1980; voir aussi

LOUKISSAS. PJ. The Impact of Tourism on Regional Development, these de doctorat non publiee, Cornell University, the Faculty of the Gra duate School, January 1977.

2. Voir L'impact du tourisme sur l'environnement, O.C.D.E. Paris, 1990:

woir aussi: COHEN, E., The Impact of Tourism on the physical environment in Annals of Tourism Research, April-June 1978, pp. 215.237.

3. Voir au sujet du multiplicateur regional fun class: que du gennel:

ISARD W. Méthodes d'analyse regionale. Dunod. Paris, 1978, pp 89110 ; pour le multiplica teur touristique:

BARETJE, R., DEFERT, P., Aspects économiques du tourisme. Berger-Levrault, Paris, 1972, pp 213222.

4. DEFERT, P. Le fourisme reflexion of mise en oes. we, in Pecherche sociale, no 48. Paris, dec. 1973 chapitre $\mathrm{IX}$

5. DEFERT, $P_{-n}$ Le tounsme rellexions et mise en oeu ve, op. cil., p. 72

6. A ce sujet woir COSSETTE, A., Le tertiarisation de l'economie quebecoise, Gaetan Morin Editeu, Chicoutimi, 1982

7. POLESE, M, Le secteur fertaire ef le developpo ment economique regional: vers un modele ope. rationnel des activites motrices, in I'Actualite Economique, wol. 50, no 4 , Montreal, 1974 , p. 476

B. Ibid, p. 479

9. DEFERT. P., Le tounsme facteur de valorisation regionale in Recherche Sociale, no 3, Paris, jan vier $1966, p .18$.

10. Ithid. p 18

11. Voir, a titre dexemple. PARENT, M. Markering Research Techniques Apohed to Tourism Planning and Development: an Example of Regression Techniques and Ski Industry Analysis, in Seminar on Travel and Tourism. ES.O.M.A.R. Stresi. 19th-22nd actober 1977 : woir auss NADEALI R AICHARD M TREMBLAY N. VIENS, A, Les joumees d'mionmation sur le tou. nisme hwemal au Quebec. Publications de I'A.T.T. no 10. Montreal, decembre 1978.

12. Voir le modele a'talement des vacances de LESAGE, J.L. MOSSE. E. Changer les vacances rapport sur l'amenagement des temps de loi sir. La Documentation Française. Paris. 1979

13. RASSI. F. Retombetes socio-économiques d'un développement touristique, M.L.C.P. Service de la recherche. Serie methodologique, vol IV. Que bec, 1975, pD. 162166, voir aussi: YOKENO, N, La localisation de Findustre tounstique: application de ranavse de Thunen-Weber, Cahiers du tourisme. Centre d'etudes du tourisme, Serie $C_{\text {. no }} 9$, Alx-enProwence, fevrier 1968; pour un resume des ditie rents modeles voir: VANHOVE. N. Forecasting an Tounsm, in Revue de tourisme, no 3. Berne, 1980

14. Voir AUGER, J., La programmation lineaire: une application dans le domaine de la récreation et du plein-air, M.LC.P. Fapport technique no 1 . Oué bec, janver 1973, pout une application au domaine du tourisme Av05SA, R, Impact tconomique du tourisme dans les Chics-Chocs. Gasptsie. MLC.P. Service de la recherche. Quebec, mars 1973

15. BRIDIER M. MICHAILOF, S., Guide pratique d'analyse de projet. Economica. Pans, 1980, p 28. voir auss Calcul teonomique et planification. La Documentation Francaige. Paris, 1973

16. CAENER, M. DOUTRIAUX J Principes d'écono mie managériale, Gaetan Morin Ed.. Chicoutimi. 1980. p. 341; woir auss. FROST, J. L'analyse coüts avantages, O.C.D.E. Paris, 1977.

17 Voir a titre diexempie. The Economic impact of Tourism Development Proposals in Niagara on the Lake, Proctor Redpern Limited, Toronto, 1980

18. Voir BARON, $Y$. Forecasting Tourism - Theory and Practice The Travel Fesearch Association, University of Utah, october 1979, pour des applications pratiques voir Halifax Citadel National Historic Park. Forecast for Use: 1979-1995. Parks Canada. Onawa, 1977: et. KARASEK, M., A Forecasting Model of Ontario Tourism, llnd Canadian Con gress on Leisure Aesearch, Toronto, 1978 